\title{
Cryptogenic fibrosing alveolitis with preserved lung volumes
}

\author{
M J Doherty, M G Pearson, E A O’Grady, V Pellegrini, P M A Calverley
}

\begin{abstract}
Background - Cryptogenic fibrosing alveolitis (CFA) is an uncommon disorder of unknown aetiology characterised by interstitial fibrosis which typically shows a restrictive pattern on pulmonary function testing. Some patients with CFA and relative preservation of lung volumes have been described and it has been suggested that their volume preservation may be due to concomitant emphysema. In a retrospective study the relative frequency of preserved lung volumes in CFA, its relationship to emphysema determined by CT scanning, its clinical features, and its subsequent natural history were investigated.
\end{abstract}

Methods - Using predefined characteristics 48 patients with CFA were identified from pulmonary function records over a three year period. Volume preservation was defined as a forced vital capacity (FVC) of $>80 \%$ predicted at presentation. Patients with relative volume preservation were compared with those with more typical pulmonary restriction and clinical data at presentation, and details of their subsequent prognosis, treatment and loss of lung function with time were obtained. Where available, computed tomographic (CT) scans for the two groups were compared in a blinded fashion to score the extent of fibrosis and the presence of concomitant emphysema.

Results - Twenty one (44\%) of the patients with CFA had an FVC of $>\mathbf{8 0} \%$ predicted. They were more likely to be male $(76 \%$ versus $48 \%$ ) and to be current smokers $(57 \%$ versus $22 \%)$ with a heavier life time cigarette consumption than the restricted patients (mean (SE) 38 (4.6) versus 25 (4.5) pack years). There were no significant differences in prognosis and subsequent treatment between the groups. Comparable HRCT scans were available in 23 subjects (seven preserved, 16 restricted). They showed no difference in extent of the pulmonary fibrosis but patients with volume preservation were more likely to show concomitant emphysema ( $86 \%$ versus $19 \%$ ). Patients with emphysema on HRCT scans were heavier smokers (41(10) versus 21(17) pack years) than those without emphysema but there was no difference in the extent of CFA score between the two groups.

Conclusions - In this area of high smoking prevalence a significant number of patients with CFA presented with relative preservation of lung volumes and $\mathrm{FEV}_{1} I$ FVC ratio. In many of these subjects this appears to reflect coincidental emphysema. This may make interpretation of gas transfer factor used to monitor progression in CFA difficult. However, there was no evidence that lung volumes at presentation were of prognostic significance. (Thorax 1997;52:998-1002)

Keywords: cryptogenic fibrosing alveolitis, lung volume, prognosis, cigarette smoking.

Cryptogenic fibrosing alveolitis (CFA) is a fibrotic disorder of the pulmonary interstitium in which pulmonary function testing typically shows a restrictive lung defect ${ }^{1-3}$ with equivalent reductions in both forced expiratory volume in one second $\left(\mathrm{FEV}_{1}\right)$ and forced vital capacity (FVC) as a percentage of predicted and decreased residual volume and transfer factor for carbon monoxide (TLCO). This results from the reduction in pulmonary compliance secondary to diffuse fibrosis. However, relative preservation of lung volumes in CFA has been reported in eight patients, all of whom were heavy smokers. ${ }^{4}$ High resolution computed tomographic (HRCT) scanning showed them to have extensive concomitant emphysema. These data were reported from a tertiary referral centre rather than a district respiratory unit and as such may have been influenced by referral bias.

Cigarette smoking has subsequently been identified as both a potential aetiological factor in $\mathrm{CFA}^{5}$ and as a predictor of decline in TLCO in this disease - an indirect marker of prognosis. ${ }^{6}$ It is not clear to what extent the effects of smoking are influenced by the presence of concomitant emphysema or whether patients with emphysema and CFA behave differently from those with CFA alone.

We hypothesised that preservation of lung volume in CFA would be associated with cigarette smoking in a dose dependent fashion and that those fibrotic patients with emphysema would follow a different natural history from those with fibrosis alone. To test these ideas we have reviewed our interstitial lung disease database over a three year period and compared the clinical, physiological and CT radiographic data between patients with preserved lung volumes and those in whom lung volumes were reduced.

Methods

All measurements of static lung volumes and transfer factor for carbon monoxide (TLCO) 
performed in our laboratory over a period of three years from June 1992 onwards were reviewed to identify cases with either clinical information suggesting fibrosing alveolitis or a low TLCo. Case notes of each potential case were obtained and those where the diagnosis of CFA was confirmed (see below) were included in the analysis. Lung volume preservation was prospectively defined as an FVC of $>80 \%$ predicted (group A, preserved volumes), patients with an FVC of $<80 \%$ being defined as restricted (group B, restricted volumes).

To be diagnosed as having CFA patients had to have the combination of fine basal inspiratory crackles with or without clubbing, a reduced TLCO, and a chest radiograph suggesting diffuse interstitial fibrosis. Patients with asbestosis, heavy occupational asbestos exposure or symptoms, signs and/or investigations suggestive of pulmonary fibrosis associated with connective tissue disorders were excluded as were those where there was a known cause of pulmonary fibrosis such as amiodarone toxicity.

CLINICAL DATA

In each confirmed case the following data were obtained: (1) sex, age at presentation, time to presentation (months) from onset of first symptoms to date of outpatient attendance; (2) symptoms categorised as breathlessness, cough, cough and breathlessness, or others; (3) self-reported exercise tolerance divided into four categories ${ }^{7}$ (<30 yards, 30-100 yards, 100200 yards, and $>200$ yards on the level); (4) smoking history in pack years; (5) presence of finger clubbing at presentation; (6) helium dilution lung volumes expressed as a percentage of predicted (ECSC normal values ${ }^{8}$ ).

CT DATA

Of the 48 patients with CFA, CT scans were available in 34 . Of these 23 were high resolution CT scans of the thorax without intravenous enhancement and were suitable for assessment of the severity of emphysema using a semiquantitative graded method. All patients except one were scanned using a GE Pace Plus CT scanner with exposure factors of $120 \mathrm{kV}$ and $130 \mathrm{~mA}$ and a scan time of three seconds during inspiration. Images were reconstructed using a bone/edge algorithm. The remaining patient was scanned using a Philips Tomoscan. All scans were reviewed retrospectively by two consultant radiologists who were unaware of the physiological data. For each patient supine and, where available (20/23), prone images were reviewed using five standard reference levels: (a) upper pole of the hilum; (b) carina; (c) lower pole of the hilum; (d) midway between (c) and (e); (e) costophrenic angle.

Scans were assessed at each level for the presence of ground glass opacification, reticular shadowing (or fibrosis), and honeycombing. Honeycombing was defined as the presence of abnormal air spaces with a wall thickness of more than $2 \mathrm{~mm}$. These changes were then used to classify the CFA throughout the lung into one of three grades: (A) ground glass appearance alone; (B) ground glass and reticular combined; (C) reticular and honeycomb appearance. ${ }^{9}$ The percentage of the level involved for each change was assessed visually and classified on a four point scale as 0 (absent), 1 ( $<25 \%$ of the area involved), 2 (25-75\% involved), or 3 (>75\% involved). An overall extent of CFA score was obtained by averaging the score for each process at each level.

Emphysema was diagnosed by the presence of areas of low attenuation, either focal unmarginated areas of low attenuation or bullae (air spaces over $1 \mathrm{~cm}$ in diameter with a wall thickness of less than $1 \mathrm{~mm}$ ). Emphysema was scored on a three point scale (mild, moderate or severe) for both low attenuation areas and for bullae at each reference level, giving a total extent of emphysema score of between 0 and 30. The extent of CFA score, the number of pack years, and the sex distribution of those subjects whose HRCT scan showed emphysema were compared with those subjects in whom the HRCT scan did not reveal emphysema. These comparisons were performed in a similar fashion to the comparisons between subjects in groups $\mathrm{A}$ and $\mathrm{B}$.

\section{SUBSEQUENT PROGRESS}

Four types of data were obtained:

(1) Treatment prescribed, if any.

(2) Group mortality. Information on any patient lost to follow up or who had not been seen in the previous six months was obtained from the patient's GP. Survival curves for both groups were constructed and compared.

(3) Change in pulmonary function with time. The FVC was used as more data points were available than for total lung capacity (TLC). The annual percentage change in percentage predicted FVC for each subject was calculated using regression analysis, regression curves were constructed for each individual and the groups were compared by comparison of the mean of the slopes.

(4) Number of subjects moving from group A to group B. No subject moved in the opposite direction.

\section{STATISTICAL ANALYSIS}

Data were expressed as mean (SD) unless otherwise stated. Comparisons between groups for categorical data were made using the $\chi^{2}$ test with $\mathrm{p}$ values of $<0.05$ being considered significant. Comparisons between continuous data were made using the Mann-Whitney U test. The prevalence of different grades of CFA and of emphysema in patients in groups $\mathrm{A}$ and $B$ was compared with the $\chi^{2}$ test and the MannWhitney U test was used to compare the extent of CFA score and the extent of emphysema score between the two groups. Survival curves were calculated using the Kaplan-Meier technique. 
Table 1 Mean (SD) pulmonary function (\% predicted) on presentation of patients with cryptogenic fibrosing alveolitis and preserved lung volumes (group $A$ ) compared with those with a restrictive defect (group $B$ )

\begin{tabular}{llll}
\hline & $\begin{array}{l}\text { Group } A \\
(n=21)\end{array}$ & $\begin{array}{l}\text { Group } B \\
(n=27)\end{array}$ & $p$ \\
\hline \% Predicted FVC & $94.2(10.5)$ & $62.6(9.4)$ & $<0.001$ \\
\% Predicted FEV & $97.9(13.4)$ & $74.4(11.4)$ & $<0.001$ \\
FEV $_{1}$ /FVC ratio & $73.2(7.4)$ & $83.2(8.9)$ & $<0.001$ \\
\% Predicted TLC & $90.1(14.6)$ & $64.7(10)$ & $<0.001$ \\
\% Predicted RV & $88.3(27.9)$ & $66.4(17.3)$ & $<0.001$ \\
\% Predicted TLCo & $46.5(12.8)$ & $53(13.4)$ & NS \\
\% Predicted KCO & $57.1(19.7)$ & $86.6(25.4)$ & $<0.05$ \\
RV/TLC\% & $34.7(6.6)$ & $37.6(7.7)$ & NS
\end{tabular}

$\mathrm{FVC}=$ forced vital capacity; $\mathrm{FEV}_{1}=$ forced expiratory volume in one second; $\mathrm{TLC}=$ total lung capacity; $\mathrm{RV}=$ residual volume; TLCO $=$ carbon monoxide transfer factor; $\mathrm{KCO}=$ carbon monoxide transfer coefficient.

\section{Results}

Of the 48 patients with CFA 21 were found to have preserved lung volumes (group A) and 27 had restricted lung volumes (group B). Initial spirometric and helium dilution lung volume data are shown in table 1 . The $\mathrm{FEV}_{1} / \mathrm{FVC}$ ratio was within the normal range in patients in group A but was significantly increased in those in group B. The mean TLCO was similarly reduced in both groups, whilst the Kco was significantly lower $(\mathrm{p}<0.005)$ in patients in group A. There was no significant difference in the RV/TLC\% between the two groups.

Clinical data (table 2) showed the ages to be similar at presentation but there were significantly more men $(16 / 21)$ in group A $(\mathrm{p}<0.02)$. These patients were more likely to be current smokers ( $57 \%$ versus $22 \%$ ) and less likely to have never smoked ( $5 \%$ versus $11 \%$ ). In the group as a whole $92 \%$ were either ex or current smokers. Life time cigarette consumption was significantly greater $(p=0.005)$ in those with preserved lung volumes and, even when non-smokers were excluded from both groups, cigarette consumption of patients in group A was still significantly greater $(\mathrm{p}=0.04)$ at 40 (19) pack years compared with 32 (22) pack years in group B.

Data on additional clinical features are given in table 3. Patients who complained of breathlessness as either their principal symptom or one of their principal symptoms were equally frequent in both groups $(71 \%$ versus $85 \%)$ but cough at presentation was significantly more common amongst those with restrictive pulmonary function (38\% versus $78 \%$ ). Some subjects presented atypically, four in group A and one in group B. These presentations were usually because of an incidental radiography report that suggested pulmonary fibrosis, al-

Table 2 Comparison of demographic factors and smoking habits between patients with cryptogenic fibrosing alveolitis and preserved lung volumes (group $A$ ) and those with a restrictive defect (group B)

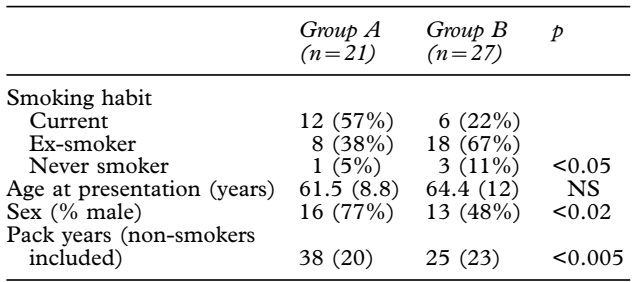

Table 3 Comparison of exercise tolerance and symptoms at presentation between patients with cryptogenic fibrosing alveolitis and preserved lung volumes (group $A$ ) and those with volume restriction (group $B$ )

\begin{tabular}{|c|c|c|c|}
\hline & $\begin{array}{l}\text { Group } A \\
(n=21)\end{array}$ & $\begin{array}{l}\text { Group B } \\
(n=27)\end{array}$ & $p$ \\
\hline \multicolumn{4}{|l|}{ Exercise tolerance } \\
\hline$<30$ yards & $2(10 \%)$ & $7(27 \%)$ & \multirow{4}{*}{ NS } \\
\hline $30-100$ yards & $4(20 \%)$ & $3(12 \%)$ & \\
\hline $100-200$ yards & $2(10 \%)$ & $1(4 \%)$ & \\
\hline$>200$ yards & $12(60 \%)$ & $15(57 \%)$ & \\
\hline \multicolumn{3}{|l|}{ Presenting symptoms } & \multirow[t]{5}{*}{$<0.05$} \\
\hline Dyspnoea & $9(42.8 \%)$ & $5(18.5 \%)$ & \\
\hline Cough & $2(9.5 \%)$ & $3(11.1 \%)$ & \\
\hline Cough and dyspnoea & $6(28.6 \%)$ & $18(66.7 \%)$ & \\
\hline Others & $4(19.1 \%)$ & $1(3.7 \%)$ & \\
\hline$\%$ clubbed & $13(62 \%)$ & $8(30 \%)$ & $<0.05$ \\
\hline \multirow{2}{*}{$\begin{array}{l}\text { Time to presentation } \\
\text { (months) }\end{array}$} & $24.2(32)$ & $194(22)$ & NS \\
\hline & & $19.4(22)$ & NS \\
\hline
\end{tabular}

though one patient had noticed the development of finger clubbing. There was no difference in self-reported exercise tolerance, but finger clubbing was more prevalent at presentation in patients in group A $(62 \%$ versus $30 \%, \mathrm{p}<0.05)$. There was no significant difference between the groups in the mean time to presentation (group A 24 (31) months versus group B 19 (23) months, $\mathrm{p}=0.4$ ).

RESULTS OF CT SCANS

Of the suitable HRCT scans seven were in patients with preserved lung volumes and 16 were in subjects in whom lung volume was restricted. The scans of all the patients in group A and 12 of those in group B had a reticular/ honeycomb appearance while the other four had reticular/ground glass changes: this difference was not significant $(p=0.15)$. There was no significant difference in the mean extent of CFA score between patients (group A 17 (8) versus group B 18 (9); $\mathrm{p}=0.4$ ).

Evidence of emphysema was seen on the CT scans of nine patients, six of whom were in group A $(p<0.001)$. Although the mean (SD) extent of the emphysema score was higher in those in group $\mathrm{A}$ than the three in group $\mathrm{B}$ (7.3 (4.6) versus $3.6(1.1)$ ), this difference was not statistically significant $(\mathrm{p}=0.1)$.

There was no difference in the extent of CFA score between those with HRCT proven emphysema and those with no emphysema (18.8 (6.7) versus $17.4(10), p=0.2)$. Eight of the nine subjects with emphysema were male compared with seven of 14 in the group without emphysema; this difference, however, was not significant $(p=0.06)$. Subjects with HRCT proven emphysema were heavier smokers (41(14) versus 21(17) pack years; $\mathrm{p}=0.004$ ). This was true even when the three never smokers were excluded from the non-emphysema group (41 (14) versus 27 (14) pack years; $\mathrm{p}=0.01)$.

\section{PROGRESS}

Nine patients (43\%) in group A received maintenance oral corticosteroids compared with 19 $(70 \%)$ in group B; this difference was not statistically significant $(p=0.06)$. Seven patients in each group were treated with oral cyclophosphamide. The mean length of follow 


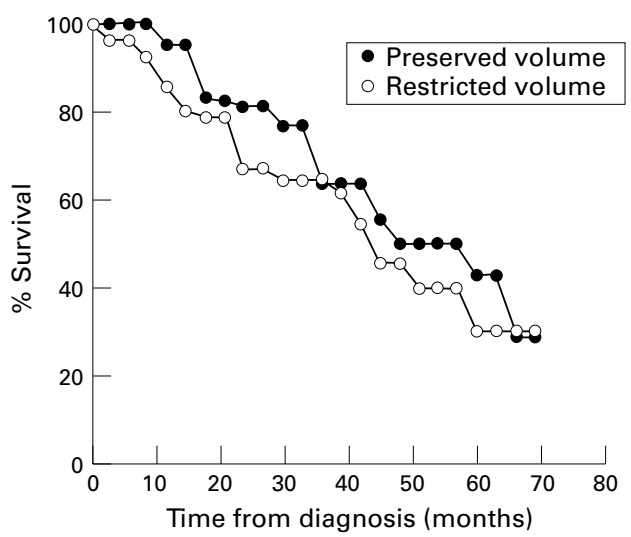

Figure 1 Survival curves of 48 patients with cryptogenic fibrosing alveolitis showing similar survival for those with volume preservation and volume restriction.

up in both groups was similar (34.8 (4.6) months for group A and 36 (8.4) months for group B) with six deaths (29\%) in group A and seven $(26 \%)$ in group B. Survival curves for both groups are presented in fig 1 and show no significant difference in survival between the two groups.

During the follow up period the FVC of six patients in group A fell below $80 \%$ predicted. When the change in FVC with time was calculated for those with follow up data of more than six months (18 in group $\mathrm{A}$ and 22 in group B) the FVC decreased by 3.95 (1.8)\% predicted per annum in patients in group $\mathrm{A}$ compared with $2.1(2.1) \%$ predicted in those in group B. This difference was not significant $(\mathrm{p}=0.3)$.

\section{Discussion}

Although previous reports of patients with CFA and preserved lung volumes have suggested that this is infrequent, ${ }^{24}$ more recent data suggest that this unlikely to be the case..$^{5610}$ Our data, which were derived from an industrial area where the prevalence of smoking is higher than in other regions of the UK, ${ }^{11}$ showed that $46 \%$ of patients had relatively preserved lung volumes. These patients were heavier smokers than those with typical restrictive problems and, given the sex difference in smoking habit, were more likely to be male. Moreover, in those with HRCT scans suitable for comparison the frequency of radiographically identifiable emphysema was higher in patients with preserved lung volumes.

Unfortunately, only 23 of the 48 subjects had comparable HRCT scans. This, and the lack of any difference in RV/TLC \% between the two groups, suggests that concomitant emphysema may not be the only cause of volume preservation. However, there is no evidence to suggest that the emphysema found on CT scanning was the result of more extensive fibrosis in these subjects, so emphysema due to smoking could explain the relative volume preservation in some cases.

Our catchment area contains a relatively stable population of 350000 and our protocol for assessment of CFA has remained the same during the last decade. The low rate of open biopsies has led us to rely on radiographic and, particularly, CT criteria for its diagnosis, a situation representative of the $\mathrm{UK}$ as a whole. ${ }^{12}$ The approximate prevalence of CFA at 14 per 100000 is in general agreement with a recent exhaustive survey from New Mexico in which 16.8 per 100000 patients were diagnosed as having idiopathic pulmonary fibrosis. ${ }^{13}$ However, our study cannot be considered to reflect accurately the true prevalence of CFA in our area as we cannot be sure that all patients with CFA were diagnosed as such. An unknown number of patients with respiratory symptoms are likely to be diagnosed as having more common respiratory diseases such as chronic obstructive pulmonary disease (COPD). In theory this underestimation might be larger in an area such as ours in which there is a high rate of respiratory symptoms and smoking.

The association between heavy cigarette smoking and emphysema verified by CT scanning in patients in group A suggests that loss of elastic recoil due to emphysema enables some lung units of high compliance to be overinflated and to empty relatively slowly. These compensate for the rapid emptying of the units of low compliance due to fibrosis and produce a composite $\mathrm{FEV}_{1}$ and $\mathrm{FEV}_{1} / \mathrm{FVC}$ ratio compatible with normal subjects. Given the high prevalence of smoking in our community this association is likely to occur by chance, although whether the fibrotic areas within the lung accelerate the development of emphysema through mechanical factors cannot be determined here.

The prevalence of breathlessness as the presenting symptom and of self-reported exercise tolerance was similar in the two groups. However, patients with preserved lung volumes were less likely to complain of troublesome cough and were more likely to have finger clubbing. Previous observations have suggested that the capsaicin cough threshold in patients with COPD is no different from that in normal subjects, ${ }^{14}$ and it is possible that the areas of lung affected by COPD reduce the overall tendency for a reduced cough threshold in CFA. ${ }^{15}$ The mechanisms underlying finger clubbing remain obscure.

Despite their apparently more favourable physiological presentation, subjects with preserved lung volumes did not show a different pattern in lung volume reduction over time nor a different mortality from patients with a typical restrictive pulmonary pattern. This is in keeping with the equivalent severity of breathlessness at presentation as breathlessness has been suggested as having prognostic significance. ${ }^{6}$ Although more of the patients with restrictive lung disease were treated, this could reflect the greater number presenting with a ground glass radiographic appearance rather than a systematic bias against patients in group A. ${ }^{16}$ The similarity in functional loss in the two groups suggests that progressive fibrosis rather than emphysema is the dominant factor here, though larger numbers would be needed to exclude a type 2 error. 
In summary, patients developing CFA in an industrial area with a high smoking prevalence often present with relative preservation of lung volumes due to the development of coincident emphysema. As a result the interpretation of potential prognostic markers such as TLCO is difficult, confirming earlier suggestions from clinicopathological studies. ${ }^{17}$ The presence of relatively normal spirometric data should not discourage the diagnosis of interstitial lung disease when other appropriate features are present.

The authors would like to thank Derek Lowe for statistical advice.

1 Turner-Warwick M, Burrows B, Johnson A. Cryptogenic fibrosing alveolitis; clinical features and their influence on survival. Thorax 1980;35:171-80.

2 Crystal RG, Fulmer JD, Roberts WC, Moss ML, Line BR, Reynolds HY. Idiopathic pulmonary fibrosis: clinical, histological, radiographic, physiological, scintigraphic, cytology and biochemical aspects. Ann Intern Med 1976; 85:769-88.

3 Austrian R, McClement JH, Renzetti AD. Clinical and physiological features of some types of pulmonary disease whysiological features of some types of pulmonary disease drome of alveolar capillary block. Am f Med 1951;11: drome $667-85$.

4 Wiggins J, Strickland B, Turner-Warwick M. Combined cryptogenic fibrosing alveolitis and emphysema the value of high resolution tomography in assessment. Respir Med 1990;84:365-9.

5 Hubbard R, Lewis S, Richards K, Johnston I, Britton J. Occupational exposure to metal or wood dust and aeti- ology of cryptogenic fibrosing alveolitis. Lancet 1996;374 $284-9$

6 Schartz DA, Van Fossen DS, Davis CS, Helmess RA Dayton CS, Burmeister LF, et al. Determinants of progression in idiopathic pulmonary fibrosis. Am $\mathcal{F}$ Respir Crit Care Med 1994;149:444-9.

7 MRC Committee on Research into Chronic Bronchitis Instructions for use of the questionnaire on respiratory symptoms. Devon: W J Holman, 1966.

8 Quanjer PH, ed. Standardised lung function testing. Bull Eur Physiopathol Respir1983;19(Suppl 15):1-95.

9 Collins CD, Wells AU, Hansell DM, Morgan RA, MacSweeney JE, Du Bois RM, et al. Observer variation in pattern type and extent of disease in fibrosing alveolitis on thin section computed tomography and chest radiography. Clin Radiol 1994;49:236-40.

10 Wells AU, King AD, Rubens MB, Cramer D, Du Bois R, Hansell DM Lone cryptogenic fibrosing alvelitis: functional-morphological correlation based on extent of disease on thin section computed tomography. Am 7 Respir Crit Care Med 1997;155:1367-75.

11 Aiming for a healthier future; a review of the health of the people in Liverpool. Liverpool Public Health Annual Report, people in Live.

12 Johnston IDA, Gomm SA, Kalra S, Woodcock AA, Evan CC, Hind CRK. The management of cryptogenic fibrosing alveolitis in three regions of the United Kingdom. Eur alveolitis in three region

13 Coultas DB, Zumwalt RE, Black WC, Sobonya RE. The epidemiology of interstitial lung diseases. Am 7 Respir Crit epidemiology of interstitial lung

14 Choudry NB, Fuller RW. Sensitivity of the cough reflex in patients with chronic cough. Eur Respir 7 1992;5:296-300.

15 Doherty MJ, Calverley PMA, Pearson MJ. Capsaicin cough test: relationship to disease and reported cough. Thora 1994;49:1069P

16 Wells AU, Hansell DM, Rubens MB, Cullinan P, Black $\mathrm{CM}$, Du Bois RM. The predictive value of appearances on thin section computed tomography in fibrosing alveolitis. Am Rev Respir Dis 1993;148:1076-82

17 Cherniack RM,Colby TV, Flint A, Thurlbeck WM, Waldron $\mathrm{JA}$, Ackerson L, et al. Correlation of structure and function in idiopathic pulmonary fibrosis. Am f Respir Crit Care Med 1995;151:1180-8. 